\title{
Philosophiques
}

\section{La théorie des systèmes luhmannienne : un nouveau paradigme en sciences sociales?}

\section{Klaus-Dieter Ertler}

Volume 21, numéro 1, printemps 1994

URI : https://id.erudit.org/iderudit/027249ar

DOI : https://doi.org/10.7202/027249ar

Aller au sommaire du numéro

Éditeur(s)

Société de philosophie du Québec

ISSN

0316-2923 (imprimé)

1492-1391 (numérique)

Découvrir la revue

Citer cet article

Ertler, K.-D. (1994). La théorie des systèmes luhmannienne : un nouveau paradigme en sciences sociales ? Philosophiques, 21(1), 3-17.

https://doi.org/10.7202/027249ar
Résumé de l'article

La présente étude se propose de repérer les traits principaux de la théorie des systèmes autopoiétiques qui a, depuis le début des années 80 , fortement marqué les discours des sciences sociales en Allemagne. Notre analyse se réfère aux concepts-clé de ce nouveau paradigme, élaboré par Niklas Luhmann, et montre leur fonctionnement. La genèse du sens telle quelle s'opère dans la société moderne se trouve au centre de l'intérèt. Avec ses trois dimensions constituantes (du temps, du social et de la chose), le sens peut être mieux observé dans l'ensemble des systèmes, qui est englobé par un environnement de plus en plus complexe. Finalement nous observerons, à titre d'essai, le système littéraire pour y relever les possibilités et les limites de cette nouvelle approche. 


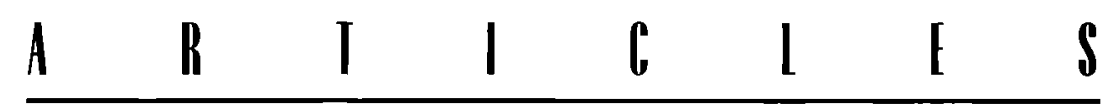

\title{
LA THÉORIE DES SYSTÈMES LUHMANNENNE : IN NOUVEAU PARADGGME EN SCIENCES SOCHALS?
}

\author{
par \\ Klaus-Dieter Ertler
}

RÉSUMÉ : La présente étude se propose de repérer les traits principaux de la théorie des systèmes autopoiétiques qui a, depuis le début des années 80 , fortement marqué les discours des sciences sociales en Allemagne. Notre analyse se réfère aux concepts-clé de ce nouveau paradigme, élaboré par Niklas Luhmann, et montre leur fonctionnement. La genèse du sens telle qu'elle s'opère dans la société moderne se trouve au centre de l'intérêt. Avec ses trois dimensions constituantes ( $d u$ temps, du social et de la chose), le sens peut être mieux observé dans l'ensemble des systèmes, qui est englobé par un environnement de plus en plus complexe. Finalement nous observerons, à titre d'essai, le système littéraire pour y relever les possibilités et les limites de cette nouvelle approche.

ABSTRACT: In the past decade, the mainstreamdiscourses in German Social Sciences have been strongly influenced by the theoretical design proposed by Niklas Luhman, which is generally called Systems Theory. In the present article I will discuss the dominant conceptions of this extremely abstract approach and focalize my reflections on the problem of the sense: How does sense evolve in social systems and how can it be observed? Is a better observation garanteed by the unfolding three dimensions of sense? An exemple like the literary system will show the 
possibilities and limits of this new paradigm in Social Sciences.

En génèral, la description ou l'auto-description d'une société ne peut pas éviter une réflexion sur son objectivité. Comme l'image visuelle d'un objet à trois dimensions exige une prise de perspective, la présentation de la réalité sociale en demande autant. Puisqu'une image sans perspective est en fait irréalisable, l'objectivité pourrait être recherchée dans une sorte de confrontation comparative des différentes structures d'aspect. Tel est pour le moins le message théorique de Karl Mannheim, un des fondateurs de la sociologie de la connaissance. Depuis les années 20 , la société - ainsi que les théories en sciences humaines - ont connu pourtant de nouvelles transformations menant à un dynamisme de plus en plus accéléré de façon à ce que, souvent, les glissements de perspectives priment sur les structures, le fonctionnalisme structural (Parsons) se fait structuralisme fonctionnel (Luhmann).

En Allemagne, un des représentants les plus en vue dans le domaine de la description de la société moderne est Niklas Luhmann ${ }^{2}$.

I. Cf. Karl Mannheim, Ideologie und Utopie (I929), Frankfurt, V. Klostermann, 1985.

2. Niklas Luhmann : Sociologue allemand généralement connu pour sa polémique avec Jürgen Habermas au début des années 70 , où il defendait le concept d'un fonctionnalisme instrumentaliste à l'encontre de l'Ecole de Francfort, selon Luhmann trop près des concepts de l'Europe ancienne, donc de la vérité, de la rationalité basée essentiellement sur la construction de l'humanisme occidental, le sujet. Convergences y a-t-il, pourtant, eu égard à l'importance de la communication et du sens.

De Luhmann, il n'y a qu'un seul ouvrage traduit en français : Amour comme passion. De la codification de lintimité, trad. par Anne-Marie Lionnet, Paris, Aubier, 1990.

D’autres publications de première importance:

- Soziologische Aufklärung, Opladen, Westdt.Verl., 1970-1987, 4 tomes.

- Theorie der Gesellschaft oder Systemrationalität, (avec J. Habermas), Frankfurt, Suhrkamp, 197I.

- Zweckbegriff und Systemrationalität, Tübingen, Mohr, 1968.

- Funktionen der Religion. Frankfurt, Suhrkamp, 1977.

- Gesellschaftsstruktur und Semantik. Studien zur Wissenssoziologie der modemen Gesellschaft, Frankfurt, Suhrkamp, 1980-1989, 3 tomes.

- Soziale Systeme, Frankfurt, Suhrkamp, 1984.

- Archimedes und wir, Berlin, Merve, 1987.

- Die Wirtschaft der Gesellschaft, Frankfurt, Suhrkamp, 1988.

- Die Wissenschaft der Gesellschaft, Frankfurt. Suhrkamp, 1992.

- Beobachtungen der Moderne, Opladen, Westdt Verl., I992. 
Depuis vingt-cinq ans, il essaie de jeter les bases d'un « design» théorique ayant plutôt référence aux fonctions des perspectives qu'à leurs normes. Par une Théorie des systèmes autopoḯtiques, il cherche à rendre accessible, aux sciences sociales en général et à la sociologie en particulier, les résultats des recherches systémiques de différentes disciplines : biologie, théorie de l'organisation, neurophysiologie, informatique, thermodynamique, théorie des cellules et autres.

La sociologie luhmannienne se fonde essentiellement sur les faits de communication pour reléguer au second plan l'activité sociale (Soziales Handeln) - doctrine-clef dans les recherches de Jürgen Habermas. Selon la version allemande de la théorie des systèmes, la question d'une société globale autarcique ne se pose plus, étant donné que l'approche se concentre sur l'autonomie des systèmes et subsystèmes ainsi que sur leur fonctionnement : Le système fonctionne à partir d'une réduction de complexité en se perdifférenciant (ausdifferenzieren) de l'environnement. Aussi peut-il être considéré à la fois comme fermé (par le code de sélection) et comme ouvert (par l'échange avec l'environnement).

Le paradigme luhmannien fonctionnant sur le dualisme système / environnement a connu, au début des années 80 , un élargissement important, à savoir l'introduction du concept de l'autopoïèse, élaboré par les neurobiologistes chiliens, H. Maturana et F. Varela. À l'aide de ce nouveau concept, la théorie peut relever tout un ensemble autopoïetique de communication qui, au lieu d'effectuer la réduction à partir de l'environnement, s'organise au moyen de ses propres éléments. Seuls ces éléments-là peuvent établir la relation à l'environnement par voie de sélection.

Cette nouvelle mèthode d'approche de la société moderne fournit des outils précieux de description : elle renonce définitivement au point d'Archimède (point de vue immuable) tant apprécié, afin de pouvoir capter les jeux et les frontières fluctuantes des perspectivisations multiples.

Il serait intéressant de revoir les causes pour lesquelles le paradigme luhmannien n'a pas eu de retombées plus importantes en France et au Québec. Bien qu'il y existe des résultats de recherches abondant dans le sens de Luhmann ${ }^{3}$ et bien que celui-ci puise dans les

3. A l'occasion de la parution de l'cuve: N. Luhmann, L'amour comme passion, Paris, Aubier 1990, Alain Gras a publie une petite introduction théorique sur la terminologie particulière de la théorie des systèmes allemande. (Cf.« Quelques mots clès de la sociologie de Niklas Luhmann », dans Cahiers intemationaux de sociologie, Vol. LXXXIX, 1990, 389-398). En particulier les travaux sur la description de la société (post)moderne de Michel Maffesoli, où les problèmes de la complexité et de l'environnement prennent une place importante : Michel Maffesoli, Le Temps des Tribus, Paris, Meridiens, 1988 ou La Connaissance ordinaire, Paris, Méridiens, 1985. 
sources françaises sans même traduire parfois les textes d'origine, le nouveau paradigme n'a pas eu droit de cité ni en France ni au Québec ${ }^{4}$. En Espagne ${ }^{5}$ et en Italie par contre, la réception ne s'y est pas fait attendre.

Nous nous proposons donc de présenter quelques idées-clef de cette sociologie de la connaissance dont les paramètres pourront fournir des instruments adéquats pour réaliser une analyse plus pertinente de la société en transformation permanente. Dans le dernier volet, nous en examinerons les conséquences à l'égard des idéologies et de la référentialité littéraire.

\section{Le sens conme élément essentiel de la théorie}

Dans sa recherche universaliste et protéiforme, afin de préciser les fonctions dirigeant les relations entre le psychique et le social, et, en outre, pour découvrir l'émergence des normes et des valeurs, Luhmann accorde une importance de plus en plus grande - après l'élimination du sujet - au concept de sens. A un tel point qu'il n'hésite pas à le tenir pour le concept de base en sociologie ${ }^{6}$. L'intérêt qu'il porte à la recherche des traces plus profondes des activités sociales l’amène à passer outre les limites constituées par le terme de « sujet » et rôdées depuis les débuts des temps modernes. Ainsi - en quête d'un terme précis pour la captation et la réduction de la contingence des mondes possibles propose-t-il le concept de sens. Le sens s'étend, entre autres, sur deux notions principales de l'œuvre théorique de Luhmann : le système psychique, fondé sur la conscience (homogène et autoréférentielle) et le

4. Les reflets de la critique systemique concernant la France ne manquent pas : la récente publication de Cornelia Bohn à propos de la théorie sociale bourdieusienne le confirme (cf. C. Bohn, Habitus und Kontext, Opladen, Westdeutscher Verlag, 1991). Selon l'auteur, Bourdieu n'attacherait pas suffisamment d'importance à la complexité sociale en insistant trop sur le fait d'un principe central de différenciation et de structuration. La hiérarchie ne peut donc pas fonctionner comme principe dominant de structuration du monde social. Le concept d'habitus - vu à partir de la théorie luhmannienne - serait trop figé pour rendre compte de la différenciation circulant dans la société moderne.

5. La monographie exhaustive sur l'œuvre luhmannien, récemment publiee en Espagne est digne d'être mentionnée : Ignacio Izuzquiza, La Sociedad sin hombres. Niklas Luhmann o la teoría como escándalo. Barcelona, Anthropos, 1990.

L'œuvre principale de N. Luhmann, Soziale Systeme, a été traduit, en espagnol sous le titre Sistemas Sociales. Lineamientos para una Teoria General, México, D.F., Alianza Editorial, r99ı.

6. Cf.N. Luhmann, «Sinn als Grundbegriff der Systemtheorie », dans J. Habermas / N. Luhmann, Theorie der Gesellschaft oder Sozialtechnologie, Frankfurt, Suhrkamp, 1985, p.25-100. 
système social, fondé sur la communication légalement homogène et autoréférentielle). Les deux systèmes s'interpénètrent, chacun représentent obligatoirement l'environnement de l'autre, sans jamais devenir indépendant. Il résulte de cet état de fait une co-évolution qui est appelée communément sens. Donner la préférence au système psychique serait de l'anthropologisme mal placé tel qu'on le connaît dans les œuvres de nos théoriciens classiques. Et il serait inconvenant de chercher le porteur de sens, car le sens se porte lui-même de par sa reproduction autoréférentielle. Ce ne sont que les formes d'une telle reproduction qui effectuent la distinction entre les structures psychiques et sociales. La conscience (système psychique) ainsi que la communication (système social) ne peuvent se créer respectivement qu'à condition de se référer à quelque chose d'autre. Le sens de son côté surgit comme conséquence d'une différence des références de sens, dont les actualisations se maintiennent par sélectivité. Le facteur principal en relation avec la fonction du sens est le foisonnement de références possibles (excédents de sens), donc le caractère contingent du monde. Par sa structure de référence, le sens exige une sélection à partir d'un champ de variétés (celui-ci peut contenir la réalité présomptive, la réalité possible et la négation sous la forme de l'irréel ou de l'impossible). Le sens fournit donc aux opérations des systèmes psychiques et sociaux la complexité requise contenant à son tour un certain degré de redondance. C'est ici que nous trouvons, à chaud, le creuset des valeurs qui se figeront - une fois la sélection effectuée-en normes et en schémas axiologiques tels qu'ils se présentent ensuite à nous, sous leur forme cristallisée en idéologie. Dans ces couches profondes, Foucault effectua de son côté des recherches sur l'épistémé ${ }^{7}$ et sur les forces énigmatiques de la genèse du discours. Luhmann s'inscrit, avec ses recherches, dans la longue liste des chercheurs qui se sont évertués à repérer les mécanismes du système de notre pensee dans les substances instables, floues, nébuleuses de la contingence et de l'entropie, tel que - à titre d'exemple - Gregory Bateson ${ }^{8}$ l'a pratiqué avec maestria.

Ce qui parait extrêmement utile dans le concept de sens, c'est son caractère fluctuant et instable dans le traitement des informations sur la base des distinctions : sa fonction consiste à former inlassablement, et chaque fois à nouveau, une différence entre actualité et possibilité. Le sens relève les possibilités au niveau de l'actualisation et se perpétue

7. Cf. M. Foucault, L'archéologie du savoir, Paris, Gallimard, 1969.

8. Cf. G. Bateson, Steps to an Ecology of Mind, San Francisco, Chandler Publishing Company, 1972. 
en ajoutant à la virtualité d'autres actualisations. Pourtant il faut remarquer avec Luhmann ${ }^{9}$, qu'aucun système producteur de sens ne pourra se soustraire au caractère sensé de ses propres processus, d'autant plus que l'ensemble des références se trouve pris dans un hermétisme circulaire, dont le dernier horizon constitue le monde (Welt). Celui-ci et le sens se présentent de la même manière inévitable et incontournable.

Revenons à la distinction de base, inhérente à tout sens, à la distinction entre le donné actuel et le donné virtuel, car c'est elle qui confère à tout « vécu » (Erleben) sa valeur d'information. Voilà un concept-clef de la théorie luhmannienne qui stipule pour tout début de processus une situation de différence au lieu d'une situation d'identité : « a difference that makes the difference », tel que Bateson ${ }^{\text {IO }}$ le propage, va dans le même sens. Il ne faut donc pas décomposer le sens en tant que différence, mais en différences. Luhmann tombe au cours de cette recherche sur trois dimensions de sens : la dimension des choses, la dimension du temps, la dimension du social ${ }^{\text {II }}$. Chacune des trois dimensions tire - de son côté - son actualité de la différence de deux horizons, donc d'une autre différence. Une telle distinction des différences sert d'une part à faire disparaître le caractère tautologique du sens, d'autre part, à introduire une respécification dimensionnelle de l'autoréférence du sens. Par la dimension des choses ${ }^{\mathrm{I2}}$, Luhmann entend la déconstruction de la structure de référence par le sens même en deux parties : « celui-ci » et « l'autre ». De façon à ce qu'il y ait articulation matérielle de sens dès qu'une disjonction première distinguant l'indéfini d'un autre indéfini s'installe (double horizon : intérieur / extérieur). Donc le premier objet de la théorie des systèmes n'est pas une chose / objet nommé système, mais bien plus au contraire la différence entre système et environnement. La constitution de la dimension $d u$ temps s'effectue par la différence avant / après et par la prolongation des événements sur deux horizons particuliers, le passé et l'avenir. Ainsi le temps devient-il libre des attaches des événements immédiats, il se fait neutre à l'égard des notions de présence / absence et trouve sa place dans la tension entre le passé et l'avenir. Le temps représente pour les

9. Cf. N. Luhmann, Sozial Systeme, op. cit., p.107.

Io. Cf. G. Bateson, op. cit., p.489, cité d'après Luhmann, ibid., p. II2.

II. Cf. N. Luhmann, « Systemtheorie, Evolutionstheorie und Kommunikationstheorie », dans : Soziologische Aufklänung, Opladen, Westdeutscher Verlag, Ig86, vol.2, p. 193-203.

I2. «Dimension concrète », selon Anne-Marie Lionnet, la traductrice d'Amour comme passion. 
systèmes de sens l'interprétation de la réalité à l'égard d'une différence entre le passé et l'avenir. Luhmann distingue pourtant deux formes de «présent »: d'une part, le présent irréversible, ponctualisé, représenté par le tic-tac de la montre, d'autre part, le présent inéluctable, permanent symbolisant en même temps le caractère réversible de tout système de sens ${ }^{13}$. Pour ce qui concerne la dimension du social, il est important de ne pas la confondre avec la dimension des choses, c'est, selon Luhmann, une faute essentielle de l'humanisme qui veut mettre le sujet ('homme) trop en valeur. Car la dimension du social met en évidence le rôle de l'alter ego sans pour autant empiéter sur le terrain de l'articulation matérielle. Dans la constitution de cette troisième dimension du sens (le social), la perspective d'un double horizon (ego / alter) compte à un tel point qu'une réduction du social au niveau d'une monade-sujet ne serait plus concevable. Conformément aux problèmes-déclencheurs des dimensions précédentes (disjonction primaire, et réversibilité / irréversibilité), on trouve ici le schématisme consensus / dissension, apte à mettre les doubles horizons de la dimension de sens sous pression d'option.

Les trois dimensions ne peuvent pas surgir de façon isolée - sauf en cas d'analyse - car elles sont soumises à la contrainte d'être combinées à tout moment à l'intérieur du sens; elles varient cependant selon la structure de la société en vigueur, donc selon le résultat de l'évolution socio-culturelle. En plus, il faut absolument rendre compte des corrélations au sein de l'appareil sémantique, constituées par les doubles horizons respectifs : intérieur / extérieur, passé / avenir, ego / alter. Pour que le sens puisse arriver à un processus autoréférentiel, il faut des généralisations symboliques ${ }^{\mathrm{I}}$ : le symbole réalise la formation de l'unité après être passé par l'opération fonctionnelle (généralisation) d'une pluralité. Luhmann ne voit pas d'autre solution pour arriver à ètablir une relation système / environnement, toute correspondance point-à-point entre les deux parties étant impossible. La généralisation (terme d'origine psychologique) représente donc l'instrument idoine, permettant de surmonter le décalage de complexité entre le système et l'environnement. Cet état de fait nous intéresse d'autant plus que la formation du concept de l'attente (Enwartung) - placé au centre de la discussion autour de la fonction idéologique en général - dépend par voie

13. Cf. N. Luhmann, Sozial Systeme, op. cit., p. I17.

14. Elles sont organisées par des codes de communication généralisés au plan symbolique, comme le sont la vérité (pour la Science), la propriété / l'argent (pour l'Économie), le pouvoir (pour le Politiquel et l'amour (pour la Famille ou I'Intimité). 
directe des « généralisations symboliques » : celles-ci produisent, par compression, une structure de référence qui a tellement de significations possibles qu'il en résulte des attentes de comportement ayant de leur côté - de l'influence par ricochet sur les généralisations. En tant que sélection, la généralisation est en même temps la limitation du possible et la présentation d'autres possibilités; les deux aspects considérés de façon synthétique (par la généralisation) suscitent l'émergence d'une complexité structurée.

\section{Système et environnement comme critères de la perdifférenniation}

Après avoir présenté le concept de sens, comme clef de voûte dans la théorie des systèmes autoréférentiels, nous présenterons de façon plus ample son paradigme essentiel : système / environnement. Les analyses fonctionnelles sans cesse proposées par Luhmann dans l'ensemble de ses articles, ne peuvent en aucun cas négliger cette distinction paradigmatique. Il faut rappeler que l'environnement n'est pas une catégorie quelconque, mais qu'il prend une place essentielle dans la formation et le maintien du système. En tant que source d'énergie et d'information, l'environnement représente en quelque sorte une autre face de la médaille, car l'identité du système ne se constitue que par différenciation avec son environnement. Cet état de fait démontre une déflagration des perspectives concernant les objets dans le monde, puisqu'il existe un manque de paramètres considérable pour, de nouveau, établir les relations de façon stable. De la même manière, les résultats d'autres analyses (complexité, sens, contrainte de sélection, double contingence) confirment - selon Luhmann ${ }^{\text {I5 }}$ - que tout ce qui existe, fait partie à la fois d'un système (ou de plusieurs) et de l'environnement d'autres systèmes. Par conséquent, tout changement d'un système entraîne l'environnement d'autres systèmes. Le fait que les personnes (systèmes psychiques) fassent partie de l'environnement des systèmes sociaux, devient donc plus justifiable et compréhensible. Et où peuton placer la réalité elle-même? Serait-elle scindée par la distinction du concept système / environnement ${ }^{16}$ ? Luhmann estime ${ }^{17}$ que la distinction de système / environnement pratiquée par un système se superpose à une réalité continue et préétablie. Tous les éléments se voient

15. Cf. N. Luhmann, Soziale System, op. cit., p. 243

16. Jean-François Lyotard veut aller plus loin que Luhmann sur cette question en introduisant la scission au sein du système même : La différence dans le discours écologique serait celle qui traverse en quelque sorte le système, le « oikos ». Extrait de « Sorge um die innere Lage », Neue Zürcher Zeitung, 16 / 10 / 1989. 
pour ainsi dire constitués - à partir d'une complexité donnée - en tant qu'unités émergeantes et ir'éductibles par le système. La complexité génératrice d'éléments fait partie de l'environnement du système, tout comme le système chimique de la cellule constitue pour le cerveau l'environnement de celui-ci, ou, de la même façon, que la conscience d'une personne représente l'environnement pour le système social. Cela n'empêche pas que le système peut établir une description de soimême (réduisant, par exemple, le système social à un contexte d'actions), indispensable à toute observation basée sur le paradigme luhmannien. Une observation interne du système social ne peut se réaliser que dans les sujets (thèmes) des processus de communication, en revanche, l'observation externe inclut des systèmes psychiques participant à la communication. Le but est d'arriver - à l'aide de la distinction système / environnement - à une observation de l'observation.

En ce qui concerne l'environnement proprement dit, il faudrait distinguer «l'environnement » de la gamme des systèmes compris dans cet environnement. Car même au niveau de la théorie générale des systèmes, la distinction entre les relations système / environnement et les relation de systèmes a été de mise. Mais tout système est devenu système par réduction de complexité (à partir de la « requisite variety ») dont le déficit trouve son équilibre dans un ordre constitué au sein du système. Luhmann insiste sur le fait que tout décalage entre le système et son environnement rend asymetrique la relation des deux côtés. Tout changement à l'intérieur du système touche nécessairement aussi le degré de complexité de l'environnement des autres systèmes. Mais le système peut rester insensible face à son environnement, ce qui lui confère une certaine autonomie dans sa propre autorégulation. Car l'émergence d'un système peut être décrite également comme une augmentation de la séléctivité pour le concret et, en même temps, comme une augmentation de l'insensibilité pour l'environnement. La régulation de la relation du système vers son environnement s'effectue donc par le mécanisme de la structure du système. Il y a par conséquent deux modes de complexité : celui de l'environnement, d'une part, et celui du système, d'autre part. En cas de trop grande complexité, le système dispose d'une structure d'orientation établie selon le paradigme et peut suivant l'exemple avancé par Luhmann ${ }^{18}$ - réagir de deux façons différentes suivant les besoins : ou bien conditionner de façon morale sa propre complexité, ou traiter celle de son entourage d'après le schéma stratégique ami / ennemi.

18. Cf. N. Luhmann, ibid., p. 251. 
Une autre réflection luhmannienne nous mène dans le domaine des fonctions des ritualisations. Elles surgissent en tant que conséquence de l'information basée sur un statut contingent et incertain de l'environnement. Les ritualisations traduisent des insécurités externes en un schématisme interne invariable jusqu'au moment où surgissent des systèmes suffisamment complexes, capables de développer - sous forme d'organisation - des équivalences fonctionnelles pour absorber l'insécurité en question.

Le décalage de complexité entre l'environnement et le système ne peut se faire qu'à condition qu'il y ait une émergence de temps au sein du système. Mais quelle relation peut-il y avoir entre le temps défini par les éléments du système et le fleuve régulier du temps dirigeant l'environnement et le système? Tant que le système respecte le mouvement régulier et continu du temps et tant qu'il se tient aux horizons du monde (horizons de passé et d'avenir valables pour l'environnement et le système), l'émergence de son propre temps à lui se produit : le temps de l'ennui, le temps restreint, le temps qu'il a à sa disposition etc. Luhmann insiste sur le fait que le développement technique détache de plus en plus l'organisation des moments temporels de la nature pour les faire ressortir à partir de problèmes de synchronisation du temps même ${ }^{19}$.

Dans la distinction globale du système et de son environnenment, il faut tenir compte de la différence entre la distinction externe (c'està-dire l'observation de l'environnement par le système) et la distinction interne (distinction du système par le système moyennant la reproduction autopoḯtique), pour mieux capter la performance des systèmes au sein de leur environnement. Par reproduction, Luhmann ne comprend nullement la répétition de la même constellation, mais une permanente reconstitution d'événements à rattacher. Et la reproduction implique à son tour la reproduction de possibilités de reproduction.

L'excédent de sens attaché à ce processus en question offre « in continenti $\gg$ la formation de nouveaux systèmes, c'est-à-dire il réalise de nouvelles distinctions, donc des restrictions au sein du système de départ et augmente de telle sorte sa complexité ${ }^{20}$.

A l'intérieur du système émergé par distinction, d'autres systèmes peuvent se former, considérant l'environnement à l'intérieur de la limite vers l'extérieur comme un environnement spécial, tout à fait réduit en complexité par rapport à l'extérieur. Tel environnement

19. Cf. N. Luhmann, ibid.,, p. 256.

20. Cf. N. Luhmann, ibid., p. 259. 
spécial fait impérativement partie du même type de système que celui qui l'englobe (par exemple les systèmes sociaux restent dans les systèmes sociaux). Le mécanisme générant les systèmes particuliers fonctionne de façon autocatalitique / autoséléctive; chacun d'entre eux constitue l'environnement pour les autres. L'éxcédant de sens (en tant que variété) mentionné plus haut stimule la sélection menant directement à la stabilisation : tel est ici, dans cette accumulation de distinctions, le point de départ pour l'evolution dans le sens darwinien du terme $^{2 \mathrm{I}}$.

Il est essentiel pour la théorie des systèmes de retenir le fait que tout système global se contient tel quel en lui-même, grâce à la reproduction autopoiétique. À titre d'exemple, il faut rappeler que Luhmann divise le système social de la « société moderne » en plusieurs systèmes fonctionnels : système fonctionnel politique, dont l'environnement est interne à la société; d'autres systèmes fonctionnels seraient l'économie, la science, la religion, l'amour ${ }^{22}$, etc. et leurs environnements respectifs internes à la même société. Dans le mouvement circulaire (la référence du système particulier au système global) s'installent pourtant des mouvements asymétriques (en tant que différence du système particulier face à son environnement, interne au système global), constitués par l'autoreproduction communicative des éléments temporalisés (événements, actions).

Les limites des systèmes sociaux, c'est-à-dire la différence entre systèmes et environnement, sont constituées - tel que nous l'avons vu plus haut - par le sens. Leur rôle est d'attribuer les éléments dont est composé un système à ce système et d'établir, par ce fait, une sorte de fonction de classement. Dans la communication, les sujets (thèmes) et les limites sont considérés comme aptes à former une cohérence bien établie. Plus que dans tout autre système, les limites des systèmes sociaux correspondent au terme de 《self-generated boundaries ${ }^{23}$. Les limites de sens s'installent en vue d'une possibilité de communication,

21. Avec la différence que la sélection de la théorie présentée ici est effectuée par le propre système, de l'intérieur, chez Darwin, au contraire, la sélection se fait par lenvironnement, de l'extérieur.

22. Á voir les nombreux travaux de l'auteur sur le code de l'amour et de la passion dans la littérature classique (française) ainsi que ses recherches sur l'émergence de l'amour / passion au cours des derniers siècles: N. Luhmann, Liebe als Passion. Zur Codierung von Intimität, Frankfurt, Suhrkamp, 1982.

23. Roger G. Barker, Ecological Psychology : Concepts and Methods for Studying the Environnement of Human Behaviour, Stanford, 1968, p. II^12, cité d'apres N. Luhmann, Sozial Systeme, op. cit., p. 269 . 
elles passent par une mutation dès que la possibilité d'une activité collective se présente.

\section{Observation et auto-observation des systèmes sociaux}

Par le renoncement au point d'Archimède, la théorie des systèmes en question représente, souple et rigide à la fois, un modèle d'interprétation de la société. La base en est la différenciation entre système et environnement, constituée de façon autopoiétique par le sens, tel que nous venons de le voir. La société moderne comprend donc plusieurs systèmes fonctionnels indépendants, qui s'observent mutuellement et continuellement.

Dans une perspective historique, cette fonctionnalisation s'est développée à partir d'un type de différenciation segmentaire (société au Moyen-Âge), pour devenir stratificatoire par la suite (société à strate supérieure figée) et pour aboutir à une différenciation fonctionnelle (société moderne). La fonctionnalisation permet, par conséquent, la réalisation de multiples observations particulières, dont les systèmes forment - de par leur schematismes binaires particuliers - les centres. Un grand avantage de cette approche est certainement la possibilité de réaliser ce que Heinz von Foerster a appelé le «second order observation $\gg^{24}$. L'observation de l'observation permet - à un niveau très abstrait - de repérer les mécanismes et les fonctions des systèmes et nous donne la possibilité de mieux suivre les aléas de la société moderne. Par l'observation de second ordre on peut ainsi «voir qu'on ne voit pas ce qu'on ne voit pas ». Certes, à ce niveau de l'abstraction, le profit en connaissance parait être minime, mais il est essentiel. Car, en observant les autres systèmes, un système peut construire des auto-références, p.ex.le système scientifique, observant actuellement s'auto-observe par l'écriture ou la lecture de cet article, en se référant à d'autres systèmes fonctionnels (économique, politique etc.). Par ce moyen, il crée les éléments dont il est composé à partir de ces mêmes éléments.

Luhmann exclut le sujet (système personnel) de l'ensemble des systèmes sociaux par le fait que l'observation ne semble être possible que dans un réseau récursif de l'observation d'observations, et non pas dans un acte singulier, subjectif ${ }^{25}$. Mais il faut bien tenir compte du fait que la distinction de l'opération et de l'observation relèvent également d'une distinction d'un observateur, ce qui mène à une paradoxie. Celleci sera finalement traitée par l'opération formelle de $《$ re-entry ${ }^{26}$,

24. Cf. Heinz von Foerster, Observing Systems, Seaside Cal., rg8I.

25. N. Luhmann, Beobachtungen der Modeme, Ffm., Opladen, 1992, p. 218.

26. Cf. George Spencer Brown, Laws of Form (1969), New York, 1979. 
développée par George Spencer Brown, où la distinction se compose de distinction et d'indication.

Les abstractions de la théorie des systèmes permettent l'observation de l'auto-description de la société et donnent ainsi un accès, qui reste indépendant des conditions historiques ainsi que des classes / intérêts sociaux. Chaque observateur constitue - par son obligation de distinguer avant d'indiquer - un monde qui reste pour lui invisible, à partir duquel il effectue ses opérations et auquel il appartient lui-même avec ses opérations. L'observateur réalise donc ses opérations à partir d'un espace non marqué. L'opération de la distinction reste également non marqué. Par cet a priori de tous les relativismes, l'analyse des idéologies circulant dans la société moderne trouvera son point de départ.

\section{Itéologie et littérature : la possibilité d'une observation}

Nos recherches portant sur les liens entre le système de la littérature avec sa référentialité littéraire et les mécanismes de l'idéologie, nous ont amené à intégrer l'approche systémique.

Nous ne sommes pas les premiers à nous poser une telle question. Loin s'en faut. Une des premières tentatives fut réalisée par Erich Köhler qui tâchait de mettre en parallèle la théorie des genres et la théorie des systèmes (selon le modèle de Luhmann) en définissant le système des genres littéraires comme moyen de « réduire la complexité de l'environnement $\gg{ }^{27}$ En prenant l'exemple du système de la poésie des troubadours, Köhler établissait une comparaison entre l'émergence autoréférentielle de systèmes partiels au sein du système social avec leur rôle de régulateur de sens, d'une part, et la fonction des genres au sein du système agissant selon les mêmes paramètres, d'autre part. Bien que le critique allemand cherchât à mettre en pratique les concepts luhmanniens de variation, contrainte d'adaptation, sélection par exclusion d'alternatives, stabilisation etc. pour rendre compte de l'évolution littéraire à l'aide du nouveau paradigme, l'homologie structurelle trop étroite entre le système social et le système litteraire qui en a résulté, a trouvé peu d'approbateurs.

La théorie des systèmes a connu un impact considérable chez les théoriciens de la réception (Jauss, Iser), dont les travaux démontrent une grande sensibilité pour le fonctionnalisme naissant qui place contrairement au structuralisme classique - le concept de fonction

27. Erich Köhler, « Gattungssystem und Gesellschaftssystem », dans Romanistische Zeitschrift fur Literaturgeschichte, Heidelberg, 1977, p. 7-8. 
devant le concept de structure, et non vice versa. C'est notamment $W$. Iser qui met en valeur le fonctionnalisme systémique par son étude sur la théorie du discours fictionnel. I'hypothèse qu'il avance fait fi de toutes les grandes théories sur la fiction (mimésis et réflexe) en conférant au texte fictionnel un rôle prépondérant dans la constitution des systèmes de sens. Dans ce contexte, le fictionnel n'est plus en opposition avec le réel, mais en communication (fonctionnelle) avec lui. La fiction contient ainsi entièrement le système de sens et peut en déceler les faiblesses ${ }^{28}$.

Enfin il ne faut pas oublier les nombreuses indications précieuses que Luhmann a données à la sociologie de la littérature. Dans un article récent ${ }^{29}$, il met au point - sous l'angle évolutionniste - la formation des époques (historiques / litteraires) à partir de l'auto-description d'un système social. Selon cette hypothèse, le système social arriverait à une sémantique plus élaborée de son auto-description par un meilleur traitement de sa propre complexité. Une autre étude, particulièrement intéressante pour notre propos porte le titre significatif « Amour comme passion. La codification de l'intimité $\gg^{3^{\circ}}$ et présente la genèse de l'individualisme et les relations intimes sous l'influence de la transition de la société à « distinction stratificatoire » à la société à «distinction fonctionnelle » vers le milieu du XVIIe siècle. Lamour y est analysé comme matière de communication, comme code de communication généralisé au plan symbolique selon les rẻgles duquel les sentiments peuvent être formés, exprimés, reniés.

Après ce bref historique des observations mutuelles entre systémisme et critique littéraire ainsi qu'idéologie, nous pouvons constater que la théorie des systèmes autopoïétiques n'a réussi à donner le coup d'envoi ni dans le domaine de l'analyse du discours (bien qu'ayant fouillé un palier plus profond que celui de la cristallisation des discours) ni dans la recherche de la fonctionnalité des idéologies.

Une conception plus fonctionnaliste des systèmes social et psychique ainsi qu'une compréhension du sens et de la réduction de complexite avec toutes ses particularités dont Luhmann a tracé les

28. Cf. Wolfgang Iser, « Die Wirklichkeit der Fiktion. Elemente eines funktionsgeschichtlichen Textmodelis der Literatur », dans R. Warning, Rezeptionsästhetik, München, UTB, 1975 .

29. N. Luhmann, « Das Problem der Epochenbildung und die Evolutionstheorie », dans H.-U. Gumbrecht $\varepsilon$ U. Link-Heer (éd.), Epochenschwellen und Epochenstrukturen im Diskurs der Literatur und Sprachhistorie, Frankfurt, Suhrkamp, I985, p. II-33.

30. Cf. N. Luhmann, Liebe als Passion. Zur Codienung von Intimität, Frankfurt, Suhrkamp, 1982. 
lignes principales pourraient servir de point de départ pour capter les processus autopoïétiques, fondés sur la reproduction évolutionnaire, et pour mieux saisir les constellations de normes et de valeurs, encore en mouvement avant leur concrétisation. La société se présente par conséquent comme système social exluant par ses limites toute complexité indéterminée et créant en même temps les structures, et partant, toutes les possibilités virtuelles qui pourraient être réalisées. L'avantage de tels procédés est que le critique se trouve juste en amont de la conception des structures, dans la mêleee des éléments flous de l'environnement dont l'un ou l'autre parvient à s'intégrer dans une relation, à se faire structure, à fonctionner dans un système.

Notre hypothèse de travail résulte de cet état de fait : Ayant l'intention de voir de plus près les liens tissés entre la littérature et la société / idéologie, il y a grand avantage à recourir à l'ensemble des systèmes d'observation ou systèmes fonctionnels. En profitant du système scientifique avec son code de communication « vérité », nous pouvons observer de quelle façon le système de l'art, en particulier celui de la littérature, effectue sa propre perdifférenciation à l'égard de l'environnement, en s'instaurant système. Ce qui nous a mené à observer de quelle façon le système en question observe - quant à lui - l'ensemble des systèmes fonctionnels de l'environnement. Par conséquent, nous avons vu au cours de nos travaux actuels, dans quelle mesure les textes littéraires d'une époque définie observent les systèmes fonctionnels de leur époque aux niveaux lexical, phrastique et transphrastique. Le système économique avec le code de communication «argent», le système politique avec le « pouvoir » ou bien le système religieux avec « la foi », pour ne nommer que les plus importants, pénètrent ainsi par voie d'observation la clôture du système littéraire. Concernant le système de la littérature, il reste encore beaucoup de cases blanches sur l'échiquier systemique : Quel code de communication prendre? Quels en seraient les schématismes binaires adéquats : forme/medium, forme / contexte, littérature / non-littérature ou bien divertissement / ennui ?

Quoi qu'il en soit, la nouvelle « objectivité » pour une critique d'idéologie ne pourra s'acquérir qu'à force de respecter l'ensemble des réseaux de sens qui circulent aux multiples niveaux des systèmes fonctionnels. Seule l'insertion de la propre perspective dans le réseau d'observations mutuelles fournira de nouveaux résultats surprenants à cette problématique. 